\title{
Robot-Assisted Distal Locking of Long Bone Intramedullary Nails: Localization, Registration, and In Vitro Experiments
}

\author{
Ziv Yaniv and Leo Joskowicz \\ School of Engineering and Computer Science \\ The Hebrew University of Jerusalem, Jerusalem 91904, Israel. \\ \{zivy, josko\}@cs.huji.ac.il
}

\begin{abstract}
We are developing an image-guided robot-based system to assist orthopaedic surgeons in performing distal locking of long bone intramedullary nails. The system consists of a bone-mounted miniature robot fitted with a drill guide that provides rigid mechanical guidance for hand-held drilling of the distal screws' pilot holes. The robot is automatically positioned so that the drill guide and nail distal locking axes coincide using a single fronto-parallel fluoroscopic X-ray. This paper describes new methods for accurate and robust drill guide and nail hole localization and registration and reports the results of our in-vitro system accuracy experiments. Tests of 17 runs show a mean angular error of $1.3^{\circ}\left(\mathrm{std}=0.4^{\circ}\right)$ between the computed drill guide axes and the actual locking holes axes, and a mean $3.0 \mathrm{~mm}$ error $(\mathrm{std}=1.1 \mathrm{~mm})$ in the entry and exit drill point, which is adequate for successfully locking the nail.
\end{abstract}

\section{Introduction}

Closed medullary nailing has become the procedure of choice for reducing fractures of the femur and the tibia [2]. It restores the integrity of fractured bone without surgically exposing the fracture with a nail inserted in the medullary canal. The surgeon reduces the fracture by percutaneously manipulating the proximal and distal bone fragments until they are aligned, inserts a guide wire into the medullary canal and drives the nail in. To prevent fragment rotation and bone shortening, the surgeon inserts lateral locking screws. The procedure is performed with a C-arm under X-ray fluoroscopy, which is used to view the position of bone fragments, surgical tools, and implants.

The insertion of the distal interlocking screws is the most challenging step of the procedure. It requires aligning the drill with the nail hole axis by repeatedly imaging the nail and drill with anterior-posterior and lateral fluoroscopic Xray images. Once the drill is aligned, drilling proceeds incrementally, with each advance verified with new images. Complications include inadequate fixation, malrotation, bone cracking, cortical wall penetration, and bone weakening due to multiple or enlarged screw holes. The surgeon's direct exposure to radiation is 3-30 minutes per procedure with $31-51 \%$ spent on distal locking alone [1]. 


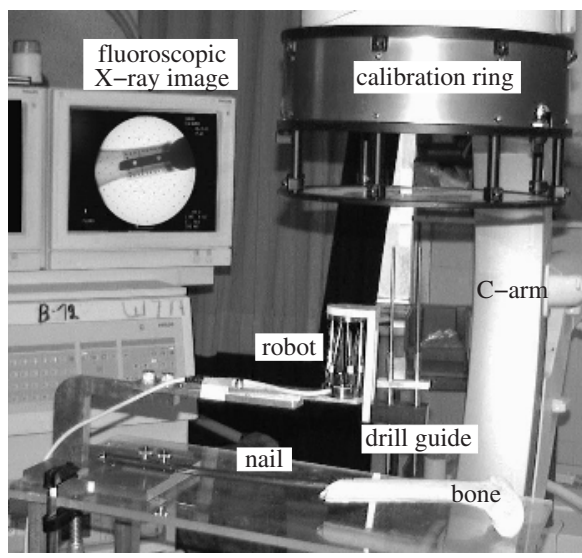

(a) In-vitro setup

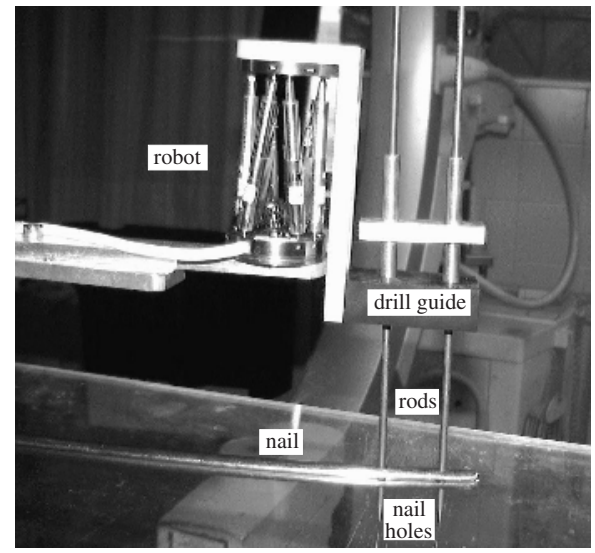

(b) aligned axes configuration

Fig. 1. Photographs of (a) the in-vitro setup and (b) the MARS robot in its desired configuration, where the drill guide and nail holes axes are aligned, as shown by the two rods passing through them.

Many devices have been developed for distal locking including proximally mounted targeting devices, mechanical guides, and stereo fluoroscopy [8]. However, all have drawbacks: they are hard to use, are not accurate enough, or cannot always be used. Surgical navigation systems [5] allow the surgeon to position and orient the hand held drill with a few augmented fluoroscopic X-ray images showing in real time the drill position. However, without a mechanical guide, they cannot prevent the drill from slipping or deviating from the desired trajectory. These drawbacks motivate our work.

\section{System Concept}

We propose to use the miniature robot MARS [10] to provide mechanical guidance for manual drilling of the holes [6]. MARS is directly mounted on the nail head or on the bone and holds a drill guide whose axes are automatically aligned with the distal nail hole axes based on a single lateral fluoroscopic X-ray image (Fig. 1). Since the robot forms a single rigid body with the bone and the nail, there is no need for leg immobilization or real-time tracking during surgery. To achieve accurate results, the fluoroscopic X-ray C-arm must be calibrated and its images corrected for distortion.

MARS is a $5 \times 5 \times 7 \mathrm{~cm}^{3}, 150$-gram 6 dof parallel manipulator whose work volume is about $10 \mathrm{~cm}^{3}$ and whose accuracy is better than $0.1 \mathrm{~mm}$. When locked, it is rigid and can withstand forces of a few kilograms. The drill guide is a Delrin block with two guiding holes $30 \mathrm{~mm}$ apart (the spacing between the nail holes). It has a pattern of $283 \mathrm{~mm}$ stainless steel fiducial spheres asymmetrically placed on two planes $20 \mathrm{~mm}$ apart that are used for its spatial localization (Fig. 2(a)). For a detailed description of MARS and the system setup see [10]. 


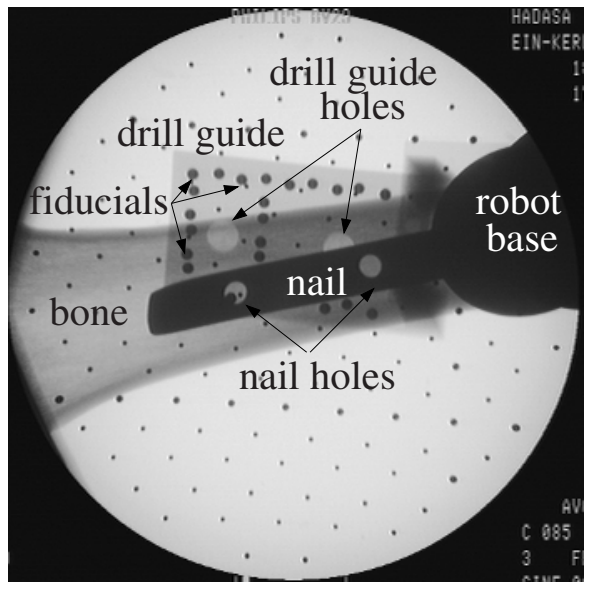

(a) before image processing

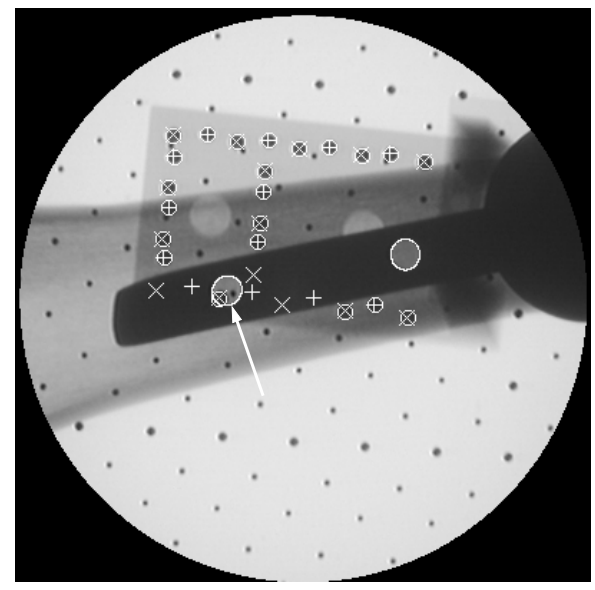

(b) after localization

Fig. 2. (a) Fluoroscopic X-ray image in fronto-parallel setting showing the robot base and drill guide, the nail and its locking holes, and the bone; (b) same image after distortion correction with the detected drill guide fiducials and pattern marked in white. The fiducials from each of the two target planes are marked as + and $\times$. The arrow indicates the correct detection of drill guide target fiducial and nail hole despite the partial occlusions.

The surgical protocol is as follows. Once the fracture has been reduced and the nail has been inserted, the surgeon mounts the robot on the nail head or on the bone. The X-ray technician mounts a calibration ring on the $\mathrm{C}$-arm image intensifier and, with the help of a guidance program, orients the C-arm so that it is in a fronto-parallel setting, where the nail holes appear as circles. The localization and registration software automatically determines from the X-ray image the locations of the drill guide and nail holes and computes the robot transformation that aligns their axes. The robot is positioned according to this transformation and locked in place. The surgeon then manually drills the holes and completes the surgery as usual.

\section{Localization and Registration}

Accurate and robust computation of the transformation that aligns the drill guide and the nail hole axes is a challenging image processing and pose estimation task. Localization of the nail holes and the drill guide is difficult because partial occlusions are inherent to the setup (the robot is mounted close to the nail holes and the image includes the nail, bone, and soft tissue). The nail holes are small $(5 \mathrm{~mm}$ diameter, about 20 pixels), nearby $(30 \mathrm{~mm})$, and appear as ellipses in the images, so the accuracy with which their axes can be determined is limited. Furthermore, only one fluoroscopic X-ray image can be used, since there 


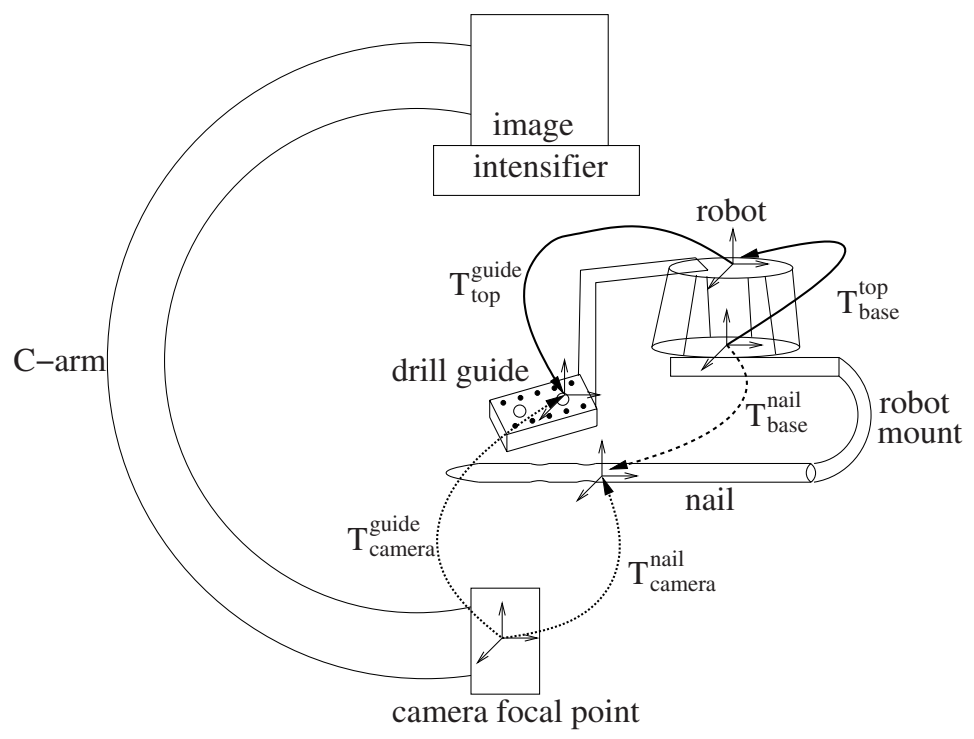

Fig. 3. Robot base to nail holes registration chain.

is no tracking of the $\mathrm{C}$-arm pose. Finally, the C-arm imaging system exhibits orientation-dependent distortions and internal imaging parameters variations.

To cope with these challenges, we have developed a novel model-based approach for robust and accurate localization of the drill guide target and nail holes and for the spatial registration of their axes. To facilitate robust nail pose estimation, we assume a fronto-parallel setup between nail and C-arm. A nearly perfect fronto-parallel pose can be obtained after a few trials by the X-ray technician with guidance from a program that scores the quality of the pose according to hole circularity and the angle between the normal of hole supporting plane and the camera viewing direction [7. When the pose is fronto-parallel, the ellipse aspect ratio is 1 (a circle) and the angle is 0 (perfect axis alignment).

We model the fluoroscopic camera as a pin-hole camera with distortion [9]. In that work, we developed a robust automatic C-arm calibration algorithm that includes fiducial localization, distortion correction, and camera calibration with an overall submillimetric accuracy even when only $60 \%$ of the fiducials are detected. The nail is modeled as a planar object with two circular holes.

To align the robot so that the drill guide axes coincide with the nail hole axes, we compute the spatial transformation between the nail and the robot base, which is given by the following transformation chain (Fig. 3):

$$
T_{\text {base }}^{\text {nail }}=T_{\text {base }}^{\text {top }} T_{\text {top }}^{\text {guide }}\left(T_{\text {camera }}^{\text {guide }}\right)^{-1} T_{\text {camera }}^{\text {nail }}
$$

where $T_{\text {base }}^{\text {top }}$ and $T_{\text {top }}^{\text {guide }}$ are known from design and $T_{\text {camera }}^{\text {guide }}$ and $T_{\text {camera }}^{\text {nail }}$ are computed from the C-arm internal camera parameters and the fluoroscopic image. 
Our method consists of four steps: 1) C-arm distortion correction and calibration; 2) drill guide identification; 3) nail hole identification; 4) drill guide and nail pose estimation. We describe the later three next.

\subsection{Drill Guide Identification}

Drill guide identification is performed by first detecting the circular fiducials in the image and then finding the correct correspondence with their 3D model. The key issues are handling partial occlusions and missing fiducials, and minimizing positional inaccuracies.

The target fiducials are detected in two steps: 1) localization and 2) circle fitting. Localization is performed using a modified circle Hough transform and a model-based analysis of the transform accumulator. Since the circular fiducials are darker than the image background, the Hough transform voting scheme is constrained so that edge pixels will only cast their vote if the vector connecting them to the hypothesized circle center is in the opposite direction of the gradient at the edge location. The contents of the transform accumulator are examined to identify the $\mathrm{k}>$ \#target fiducials which received the most votes. Considering a few more candidate circles is necessary since the accumulator may contain multiple peaks for the same fiducial (these are higher than the peaks for other fiducials). The algorithm then computes the average radius and number of votes of the five circles with the most votes and selects all circles whose radius is within \pm 2 pixels of the average radius and which have received more than half of the average votes. These circles, with very high probability, belong to the target.

The selected set of circles may contain overlapping circles, which are due either to multiple responses for the same fiducial or to fiducial overlap. In our imaging setup, there can be at most two overlapping fiducials. Thus, for each set of overlapping circles, we only retain the two circles with the highest number of votes. These pairs of overlapping circles correspond either to two overlapping fiducials or to a single fiducial. When the overlap area between the pair of circles is larger than $60 \%$ of the circle's area, it is a single circle, otherwise it is two. Circle fitting is performed using the Random Sample Consensus paradigm [3]. For each circle, the algorithm collects all edge elements contained in its circumscribing square and fits a circle to them. This removes the dependency on the Hough transform discretization accuracy.

The correspondence between the detected fiducials and their $3 \mathrm{D}$ model is computed using homographies. Correctly pairing the detected fiducials and the $3 \mathrm{D}$ model fiducials is difficult, as there are always missing fiducials which are occluded by the nail. To overcome these problems, we use the geometry of the drill guide target: we use lines instead of points, since lines are less sensitive to partial occlusions.

Since the fiducials are distributed on two planes, the goal is to find the pair of homographies which minimizes the distance between the detected circles and the result of applying the homographies to the target fiducials. The pair of homographies is computed in three steps. First, we identify the line which passes through the maximal number of detected circles. This line is one of the 
pattern axes. Next, we find all lines which are parallel or perpendicular to this line (within $\pm 5^{\circ}$ ) and that pass through two circles only. The target is built such that the two spheres on these lines are on the same plane. Finally, the best match among the small set of possible lines is selected by trying all combinations. The target fiducials are then mapped with the homographies to the image and the detected circles are identified accordingly (Fig. [2 b)).

\subsection{Nail Hole Identification}

Distal locking nail holes identification is performed by first locating the nail's longitudinal contour and then locating its holes from their expected position with respect to this contour.

To locate the nail longitudinal contours, we use a 3D Hough transform in which the nail is modelled as a band consisting of two parallel lines at a known distance between them. The Hough transform voting scheme is constrained so that pixels which are on parallel lines will only cast their vote if the gray level values between them are lower than the gray level values outside the band. All the pixels outside the contours are discarded.

The search for the nail holes is then performed on the pixels contained between the nail's contours. The algorithm sweeps a parallelepiped window whose sizes are equal to the nail width along the nail's medial axis. The two locations containing the maximal number of edge elements correspond to the locations of the distal locking nail holes. The algorithm then fits an ellipse to the edge data contained inside the parallelepipeds. The edge elements originate from the nail holes, the drill guide target and the C-arm calibration target (Fig. 2(b)). The ellipse parameter estimation is performed by using only the edge elements which belong to the convex hull of the set of elements. This is because ellipses are convex shapes and because the nail is opaque, so outlying edges can only be present in the interior of the ellipse. Next, an ellipse is fitted to the edge elements with a non-linear geometric least squares method initialized with the estimate computed using an algebraic least squares method [4].

\subsection{Drill Guide and Nail Pose Estimation}

The drill guide pose is computed by non-linear minimization of the projection distances between the known fiducial projection coordinates $\left(x_{i}, y_{i}\right)$ and the expected ones $\left(\widehat{x_{i}}, \widehat{y_{i}}\right)$ :

$$
\mathbf{v}^{*}=\underset{\mathbf{v}}{\arg \min } 0.5\left(\sum_{i=1}^{n}\left(x_{i}-\widehat{x}_{i}(\mathbf{v})\right)^{2}+\left(y_{i}-\widehat{y}_{i}(\mathbf{v})\right)^{2}\right)
$$

where $\mathbf{v}$ is the rigid transformation parameterization. We use the LevenbergMarquardt method with an initial estimate obtained with a linear method [1].

The nail pose is computed using the fronto-parallel setup assumption, in which the projective transformation applied to the nail holes is a similarity. The 
pose is computed directly from the nail holes coordinates in the image, $\left(\mathbf{p}_{x}, \mathbf{p}_{y}\right)$ and $\left(\mathbf{q}_{x}, \mathbf{q}_{y}\right)$, their average diameter on the image $d_{i m}$, their real diameter $d$, and the camera focal length $f$. The nail location is:

$$
\mathbf{t}=\left[\begin{array}{c}
\frac{z}{f} \mathbf{p}_{x} \\
\frac{z}{f} \mathbf{p}_{y} \\
z
\end{array}\right]
$$

where $z=f d / d_{i m}$ is the nail distance from the camera focal point. The nail orientation relative to the camera is:

$$
R=\left[\begin{array}{ccc}
\mathbf{p}_{x}-\mathbf{q}_{x} & \mathbf{p}_{y}-\mathbf{q}_{y} & 0 \\
\mathbf{p}_{y}-\mathbf{q}_{y} & \mathbf{q}_{x}-\mathbf{p}_{x} & 0 \\
0 & 0 & -1
\end{array}\right]
$$

The nail's $X$ axis direction depends on the order in which $(\mathbf{p}, \mathbf{q})$ are chosen. It is set so that its angular deviation from the drill guide's $X$ axis is minimal.

\section{Experimental Results}

We conducted three sets of experiments to quantify the accuracy and robustness of the proposed method. To test the robustness of the drill guide and nail hole identification, we manually placed the robot and $\mathrm{C}$-arm in random positions. We then acquired 67 fluoroscopic X-ray images. The drill guide and nail holes were correctly identified in 61 images. There were no false positives, i.e., the detection was correct in all images for which our software stated that the drill guide and nail holes were detected. All six failures were automatically detected by the software. They were caused by occlusion of many drill guide target fiducials, which prevented its proper localization.

To quantify the accuracy of the whole system for the task at hand, we define two error measures: the angular deviation of the drill guide axes and the distance between axes entry and exit points, which is the in-plane distance between the intersection points of the axes and two planes located at $100 \mathrm{~mm}$ and $120 \mathrm{~mm}$ from the robot base (the nail is located between these planes).

Next, the robot was manually placed in a pose in which two $4 \mathrm{~mm}$ diameter cylindrical rods with tapered ends pass through the $5 \mathrm{~mm}$ diameter drill guide and nail holes as shown in Fig. 1(b). This pose guarantees successful locking and constitutes the robot's reference pose. To quantify the variability of the reference poses, we placed the robot in 17 different poses in which the two rods passed through the drill guide and nail holes and computed the two error measures. We found an angular variation of $0^{\circ}-0.9^{\circ}$ and a translational variation in the entry and exit points of $0-3.9 \mathrm{~mm}$. This means that a registration accuracy of about $1^{\circ}$ and $\pm 2 m m$ will guarantee successful distal locking.

Next, under the guidance of our software, we oriented the C-arm so that it formed a fronto-parallel setup with the nail holes. We placed the robot in 17 random poses, acquired fluoroscopic X-ray images for each pose, and computed 
the robot alignment transformation for each pose (in all cases, the drill guide target and nail holes were correctly detected). Comparing the 17 computed robot poses to the reference pose our results show a mean angular error of $1.3^{\circ}$ (std = $\left.0.4^{\circ}\right)$ between drill guide axes, and a mean $3.0 \mathrm{~mm}$ error $($ std $=1.1 \mathrm{~mm}$ ) in the entry and exit points, which is adequate for successfully locking the nail.

\section{Conclusions}

We have presented a robust, automatic method for aligning the drill guide and the nail holes axes based on a few fluoroscopic X-ray images. The method is part of a new image-guided miniature robot-based system to assist orthopaedic surgeons in performing distal locking of long bone intramedullary nails. Our experimental results show that the automatic localization is feasible within the required accuracy. We are currently working on mechanical and algorithmic improvements to increase the accuracy of the system.

\section{References}

1. Ansar A., Daniilidis K., "Linear pose estimation from points or lines", IEEE Trans. on Pattern Analysis and Machine Intelligence, 25(5), 2003.

2. Brumback R.J., "Regular and special features - the rationales of interlocking nailing of the femur, tibia, and humerus", Clinical Orthopaedics, 324, 1996.

3. Fischler M. A., Bolles R. C., "Random sample consensus: a paradigm for model fitting with applications to image analysis and automated cartography", Communications of the ACM, 24(6), 1981.

4. Fitzgibbon A., Pilu M., Fisher R. B., "Direct least square fitting of ellipses", IEEE Trans. on Pattern Analysis and Machine Intelligence, 21(5), 1999.

5. Joskowicz L., Hazan E., "Computer-assisted image-guided intramedullary nailing surgery of femoral fractures" (in French), Monographie des Conferences d'Enseignement de la SOFTCOT, Vol. 80, Elsevier, 2003.

6. Joskowicz, L., Milgrom, C., Shoham, M., Yaniv, Z., Simkin, A., "A robot-assisted system for long bone intramedullary distal locking: concept and preliminary results", Proc. of the 17th Int. Congress on Computer-Assisted Radiology and Surgery, CARS'2003, H.U. Lemke et. al. editors, Elsevier 2003, pp 485-491.

7. Kanatani K., Liu W., "3D interpretation of conics and orthogonality", Image Understanding, $\mathbf{5 8}(3), 1993$.

8. Krettek C. et al., "A mechanical distal aiming device for distal locking in femoral nails", Clinical Orthopaedics, 384, 1999.

9. Livyatan H., Yaniv Z., Joskowicz L., "Robust automatic C-arm calibration for fluoroscopy-based navigation: a practical approach", Proc. of Medical Image Computing and Computer Assisted Intervention, 2002.

10. Shoham, M., Burman, M., Zehavi, E., Joskowicz, L., Batkikin, E., Kunicher, Y., "Bone-mounted miniature robot for surgical procedures: concept and clinical applications", IEEE Trans. on Robotics and Automation, 19(5), 2003.

11. Skjeldal S., Backe S., "Interlocking medullary nails - radiation doses in distal targeting", Archives of Orthopaedic Trauma Surgery, 106, 1987. 